\title{
NEAR-RINGS ASSOCIATED WITH MATCHED PAIRS ON RING MODULES
}

\author{
C. J. MAXSON AND A. P. J. VAN DER WALT
}

(Communicated by Lance W. Small)

\begin{abstract}
Let $G$ be a module over a ring $R$, let $\mathscr{C}=\left\{C_{i}\right\}, i \in I$, be a family of submodules of $G$, and let $\mathscr{H}=\left\{H_{i}\right\}, i \in I$, where $H_{i}$ is a subgroup of $\operatorname{Hom}_{R}\left(C_{i}, G\right)$ with certain properties. To each such pair $(\mathscr{C}, \mathscr{H})$, a nearring $M(\mathscr{C}, \mathscr{H})$ is associated, which is a generalization of the near-ring of homogeneous functions determined by $(G, R)$. The transfer of information from module properties of $G_{R}$ reflected in $(\mathscr{C}, \mathscr{H})$ to structural properties of $M(\mathscr{C}, \mathscr{H})$ is investigated.
\end{abstract}

\section{INTRODUCTION AND BASIC CONCEPTS}

Let $R$ be a ring with identity, and let $G$ be a unitary right $R$-module. Then, under function addition and function composition, the set $M_{R}(G):=\{f: G \rightarrow$ $G \mid f(a r)=f(a) r, \forall a \in G, \forall r \in R\}$ is a zero-symmetric near-ring with identity, called the near-ring of homogeneous functions determined by the pair $(R, G)$. The ring $S:=\operatorname{End}_{R} G$ is a subring of $M_{R}(G)$. The near-rings $M_{R}(G)$ have been the subject of several investigations. (See [2] and the references given there.) Recently, sub-near-rings of $M_{R}(G)$ in which the homogeneous functions can be represented locally as endomorphisms of $G$ have been investigated. We proceed to define these subrings.

Let $\mathscr{C}=\left\{C_{\alpha}\right\}, \alpha \in \mathscr{A}$, be a family of submodules of $G$ with the properties: (i) $\bigcup \mathscr{C}=\bigcup_{\alpha \in \mathscr{A}} C_{\alpha}=G$, and (ii) for each $s \in S$ and each $C_{\alpha} \in \mathscr{C}$, there exists $\beta \in \mathscr{A}$ such that $s\left(C_{\alpha}\right) \subseteq C_{\beta}$. In this case we say $\mathscr{C}$ is a cover for $G$. For each cover $\mathscr{C}$ of $G$ we have a sub-near-ring $P E_{R}(G, \mathscr{C})$ of $M_{R}(G)$ given by $P E_{R}(G, \mathscr{C}):=\left\{f \in M_{R}(G)|f| C_{\alpha}\right.$ extends to some $\left.s \in \operatorname{End}_{R} G, \forall \alpha \in \mathscr{A}\right\}$, called the near-ring of piecewise endomorphisms of $G_{R}$ determined by $\mathscr{E}[3$, 4]. In [3] it was shown that for finitely generated modules $G$ over a principal ideal domain $D$, if one uses the cover $\mathscr{P}$ of cyclic submodules then $M_{D}(G)=$ $P E_{D}(G, \mathscr{P})$, i.e., every homogeneous function is locally an endomorphism of $G$. It is not known if this characterizes finitely generated modules over principal ideal domains.

It is the purpose of this work to initiate an investigation of a generalization of near-rings of piecewise endomorphisms. The main goal of our program of study

Received by the editors September 2, 1992 and, in revised form, February 8, 1993.

1991 Mathematics Subject Classification. Primary 16Y30; Secondary 16D10. 
is to develop concepts and techniques which may then be applied to near-rings of piecewise endomorphisms to obtain a better understanding of this situation.

We now present the basic definitions and concepts for our work. Throughout the paper, all rings $R$ will have an identity and all $R$-modules $G$ will be unitary.

Let $\mathscr{C}=\left\{C_{i}\right\}, i \in I$, be a family of submodules of $G$, where $I$ is an index set and $C_{i} \neq C_{j}$ when $i \neq j$. Let $\mathscr{H}=\left\{H_{i}\right\}, i \in I$, be a family with $H_{i}$ subgroups of $\operatorname{Hom}_{R}\left(C_{i}, G\right)$. The pair $(\mathscr{C}, \mathscr{H})$ is called a matched pair on $G$ if: (a) $f \in H_{i}$ implies $f\left(C_{i}\right) \in \mathscr{C}$, and (b) $f \in H_{i}$ with $f C_{i}=C_{j}$ and $g \in H_{j}$ implies $g f \in H_{i}$.

In this definition, as in the sequel unless otherwise stated, unquantified indices like $i$ and $j$ will be understood to range freely over $I$. We shall find it useful to let $H:=\bigcup \mathscr{H}$ and to define the functions $\sigma, \tau: H \rightarrow I$ by $\sigma(h)=i$ if $h \in H_{i}$ and $\tau(h)=j$ if $h C_{\sigma(h)}=C_{j}$. This is well defined because $C_{i} \neq C_{j}$ when $i \neq j$. Also in the sequel we assume without specific mention that we have some matched pair $(\mathscr{C}, \mathscr{H})$ with accompanying index set $I$.

Associated with each matched pair $(\mathscr{C}, \mathscr{H})$ on $G$ is a near-ring $M:=$ $M(\mathscr{C}, \mathscr{H})$ which we proceed to define. For our set $M$ we take $M=\times_{I} H_{i}=$ $\left\{\left\{s_{i}\right\}, i \in I \mid s_{i} \in H_{i}\right\}$. Addition on $M$ is defined to be componentwise, and multiplication on $M$ is defined for $s, t \in M$ by $(s t)_{i}:=s_{\tau\left(t_{i}\right)} t_{i}$.

Theorem I.1. $M=M(\mathscr{C}, \mathscr{H})$ is a zero-symmetric abelian near-ring with the property $s(-t)=-(s t), \forall s, t \in M$.

Proof. Clearly $(M,+)$ is an abelian group. We verify right distributivity and associativity. Right distributivity follows from

$$
\begin{aligned}
{[(s+t) u]_{i} } & =(s+t)_{\tau\left(u_{i}\right)} u_{i}=\left(s_{\tau\left(u_{i}\right)}+t_{\tau\left(u_{i}\right)}\right) u_{i} \\
& =s_{\tau\left(u_{i}\right)} u_{i}+t_{\tau\left(u_{i}\right)} u_{i}=(s u)_{i}+(t u)_{i}
\end{aligned}
$$

and associativity from

$$
\begin{aligned}
{[s(t u)]_{i} } & =s_{\tau\left((t u)_{i}\right)}(t u)_{i}=s_{\tau\left(t_{\tau\left(u_{i}\right)}\right)} t_{\tau\left(u_{i}\right)} u_{i} \\
& =(s t)_{\tau\left(u_{i}\right)} u_{i}=[(s t) u]_{i} .
\end{aligned}
$$

Finally, if $h, k \in H$ and $h k$ is defined, then $h(-k)=-h k$. This completes the proof.

Corollary I.2. If $1_{C_{i}} \in H_{i}$ for all $i \in I$, then $M$ is a near-ring with identity $e$, where $e_{i}:=1_{C_{i}}$.

Unless stated to the contrary we always assume $1_{C_{i}} \in H_{i}$ for all $i \in I$. This implies that $\varepsilon^{i} \in M$ where $\left(\varepsilon^{i}\right)_{j}:=0$ if $j \neq i$ and $\left(\varepsilon^{i}\right)_{i}=e_{i}$.

Theorem I.3. The $\varepsilon^{i}$ are mutually orthogonal.

Suppose $\mathscr{P}=\left\{C_{i}\right\}, i \in I$, is the collection of all cyclic submodules of an $R$-module $G$. If we let $\mathrm{Hom}:=\left\{H_{i}\right\}, i \in I$, where $H_{i}:=\operatorname{Hom}_{R}\left(C_{i}, G\right)$, then $(\mathscr{P}$, Hom $)$ is a matched pair on $G$. However, $M(\mathscr{P}$, Hom $)$ does not necessarily represent a near-ring of functions on $G$ since, for $s \in M(\mathscr{P}$, Hom $)$ and $x \in C_{i} \cap C_{j}, s_{i} x$ may be different from $s_{j} x$. Consequently we define $H F(\mathscr{P}$, Hom $):=\left\{s \in M\left|s_{i}\right| C_{i} \cap C_{j}=s_{j} \mid C_{i} \cap C_{j}, \forall i, j \in I\right\}$. Straightforward calculations show that $H F(\mathscr{P}$, Hom $)$ is a sub-near-ring of $M(\mathscr{P}$, Hom $)$. Then $H F(\mathscr{P}, \mathrm{Hom})$ can be thought of as a near-ring of functions of $G$ by 
defining $s(g)=s_{i} g$ if $g \in C_{i}$ and $s \in H F(\mathscr{P}$, Hom). Moreover, since $s_{i} \in \operatorname{Hom}_{R}\left(C_{i}, G\right)$, we have $s(g r)=(s g) r$, for each $r \in R$. So, we have a near-ring of homogeneous functions on $G$. A sub-near-ring, $P E(\mathscr{P}, \mathrm{Hom})$ of $H F(\mathscr{P}, \mathrm{Hom})$ is defined by $\operatorname{PE}(\mathscr{P}, \mathrm{Hom}):=\left\{s \in H F(\mathscr{P}\right.$, Hom $)\left|s_{i}=\rho\right|_{C_{i}}$ for some $\left.\rho \in \operatorname{End}_{R} G\right\}$. This is a near-ring of piecewise endomorphisms on $G$ which we denoted previously by $P E_{R}(G, \mathscr{P})$. Thus, in this sense, our nearrings $M(\mathscr{C}, \mathscr{H})$ are indeed generalizations of the near-rings of piecewise endomorphisms.

For $h, g \in H$, if $h g$ is defined, then one has $\sigma(h g)=\sigma(g)$ and $\tau(h g)=$ $\tau(h)$. Now let $s \in M$, and define $\operatorname{suppt}(s)=\left\{\sigma\left(s_{i}\right) \mid s_{i} \neq 0\right\}, \operatorname{null}(s)=$ $\left\{\sigma\left(s_{i}\right) \mid s_{i}=0\right\}$, and target $(s)=\left\{\tau\left(s_{i}\right) \mid i \in I\right\}$. We then have the following lemma, whose proof is left to the reader.

Lemma 1.4. For $s, t \in M$,

(i) $\operatorname{suppt}(s t) \subseteq \operatorname{suppt}(t)$,

(ii) $\operatorname{null}(s t) \supseteq \operatorname{null}(t)$,

(iii) $\operatorname{target}(s t) \subseteq \operatorname{target}(s)$, and

(iv) $|\operatorname{target}(s t)| \leq|\operatorname{target}(t)|$.

As an application of the above we take $R=\mathbb{Z}$ and $G=\mathbb{Z}_{\mathbb{Z}}$ and let $\mathscr{C}_{\mathbf{Z}}=\left\{C_{i}\right\}$ be the set of all ideals of $\mathbb{Z}$ where the indexing set $I=\mathbb{N}_{0}$ and $C_{i}=\mathbb{Z} i$, the ideal generated by $i$. Further let $H_{i}:=\operatorname{Hom}_{\mathbf{Z}}\left(C_{i}, \mathbb{Z}\right)$.

Lemma I.5. If $s, t \in M\left(\mathscr{C}_{\mathbb{Z}}, H\right)$ are such that $|\operatorname{target}(s)|<\infty$ and $|\operatorname{target}(t)|<$ $\infty$, then $|\operatorname{target}(s+t)|<\infty$.

Proof. Define an equivalence relation $\sim$ on $I$ by $i \sim j$ if and only if $\tau\left(s_{i}\right)=$ $\tau\left(s_{j}\right)$ and $\tau\left(t_{i}\right)=\tau\left(t_{j}\right)$. By hypothesis, $\sim$ has finitely many equivalence classes. Let $J$ be one such class. We show that if $i \in J$, then there are just finitely many possibilities for $\tau\left((s+t)_{i}\right)$. If $\tau\left(s_{i}\right)=j$ then $s_{i}(i)=j$ or $-j$, and likewise if $\tau\left(t_{i}\right)=k$ then $t_{i}(i)=k$ or $-k$. Hence $\tau\left((s+t)_{i}\right)=| \pm j \pm k|$.

Recall that an additive subgroup $A$ of an arbitrary near-ring is invariant if $N A \subseteq A$ and $A N \subseteq A$. If the only invariant subgroups of $N$ are $\{0\}$ and $N$, we say $N$ is invariantly simple. The previous two lemmas show that $\left\{s \in M\left(\mathscr{C}_{\mathbf{Z}}, \mathscr{H}\right)|| \operatorname{target}(s) \mid<\infty\right\}$ is a nontrivial proper invariant subgroup of $M\left(\mathscr{C}_{\mathrm{Z}}, \mathscr{H}\right)$.

Theorem I.6. $M\left(\mathscr{C}_{\mathbf{Z}}, \mathscr{H}\right)$ is not invariantly simple.

We remark that this theorem can be generalized to rings in which each nonzero element has a finite number of associates, e.g. $\mathbb{Z}[x], \mathbb{Z}\left[x_{1}, \ldots, x_{n}\right]$, and $F\left[x_{1}, \ldots, x_{n}\right]$ where $F$ is a finite field.

On the other hand we now show that $M\left(\mathscr{C}_{Z}, \mathscr{H}\right)$ is a simple near-ring. We note that this also follows from subsequent results.

Theorem I.7. $M\left(\mathscr{C}_{\mathrm{Z}}, \mathscr{H}\right)$ is a simple near-ring.

Proof. We adopt the following convention. If $s \in M\left(\mathscr{C}_{\mathrm{Z}}, \mathscr{H}\right), s_{i}=k$ will mean $s_{i}(i)=k$. Now suppose $s \neq 0$, say $s_{m}=k$. We may assume $k>0$; otherwise use $-s$. Let $x$ and $y$ be defined by $x_{i}=m, y_{i}=1$ for $i>0$. Then $(y s x)_{i}=1$ for $i>0$. Now let $u$ and $v$ be defined by $u_{i}=i-1$, $v_{i}=i(i+1) / 2, i>0$. Then $y s x+u=e, v(y s x+u)=v$, and $(v u)_{i}=v_{i-1}$, 
$i>0$. Hence $[v(y s x+u)-v u]_{i}=v_{i}-v_{i-1}=i, i>0$, so $e$ is in the ideal generated by $s$.

We now present a very general way of constructing matched pairs on an $R$-module $G$. To this end let $\mathscr{A}$ be any class of right $R$-modules, and set

$$
\mathscr{C}_{G}(\mathscr{A}):=\left\{\operatorname{Im} f \mid f \in \operatorname{Hom}_{R}(A, G), A \in \mathscr{A}\right\} .
$$

If $\mathscr{A}=\{A\}$, we write $\mathscr{C}_{G}(A)$ for $\mathscr{C}_{G}(\{A\})$. Suppose $\mathscr{C}_{G}(\mathscr{A})=\left\{C_{i}\right\}, i \in I$, where this indexing is done in such a way that $C_{i} \neq C_{j}$ when $i \neq j$. Two families of sets of mappings are natural candidates to be the second member of the matched pair, viz. Hom $:=\left\{\operatorname{Hom}_{R}\left(C_{i}, G\right)\right\}, i \in I$, and End $:=\left\{\left\{f\left|C_{i}\right| f \in\right.\right.$ $\left.\left.\operatorname{End}_{R} G\right\}\right\}, i \in I$. However, there are many other possibilities, e.g., if $T$ is any subring of $\operatorname{End}_{R} G$, then $\left\{\left\{f\left|C_{i}\right| f \in T\right\}\right\}, i \in I$, is a candidate for $\mathscr{H}$. We note that if $(\mathscr{C}$, Hom $)$ is a matched pair, then $\mathscr{C}=\mathscr{C}_{G}(\mathscr{C})$. The next result states a number of obvious facts about matched pairs.

Theorem I.8. (a) Let $(\mathscr{C}, \mathscr{H})$ and $\left(\mathscr{C}^{\prime}, \mathscr{H}^{\prime}\right)$ be matched pairs on $G$. If $\mathscr{C} \subseteq$ $\mathscr{C}^{\prime}$ and $H \subseteq \mathscr{H}^{\prime}$, then $M(\mathscr{C}, \mathscr{H})$ is a sub-near-ring of $M\left(\mathscr{C}^{\prime}, \mathscr{H}^{\prime}\right)$.

(b) $\mathscr{C}_{G}(R)$ is the family of all cyclic submodules of $G$.

(c) $\mathscr{C}_{G}(\mathscr{S})$, where $\mathscr{S}$ is the class of simple $R$-modules, is the family of simple submodules of $G$.

Let $(\mathscr{C}, \mathscr{H})$ be a matched pair on $G$ with relevant indexing set $I$. We define a relation $\sim$ on $I$ by $i \sim j$ if there exist $h, k \in H$ with $\sigma(h)=\tau(k)=i$ and $\tau(h)=\sigma(k)=j$. It is straightforward to verify that $\sim$ is an equivalence relation. We denote the set of equivalence classes by $I / \sim$ and represent an equivalence class of $\sim$ by $[i]$. We next define a relation $\preceq$ on $I / \sim$ by $[i] \preceq[j]$ if there exists an $h \in H$ with $\sigma(h) \in[j]$ and $\tau(h) \in[i]$. Using the definition of matched pair one verifies that $\preceq$ is a partial order on $I / \sim$.

Theorem I.9. If every $h \in H_{i} \cap \operatorname{End}_{R} C_{i}$ is an automorphism of $C_{i}$, then every $h$ with $\sigma(h) \in[i]$ and $\tau(h) \in[i]$ is an isomorphism.

Proof. If $\sigma(h), \tau(h) \in[i]$, then there exist $h_{1}, h_{2} \in H$ with $\sigma\left(h_{1}\right)=i$, $\tau\left(h_{1}\right)=\sigma(h), \sigma\left(h_{2}\right)=\tau(h)$, and $\tau\left(h_{2}\right)=i$. This implies that $h_{2} h h_{1}$ is an endomorphism of $C_{i}$ and, so by hypothesis, an automorphism. But this in turn implies $h$ is an isomorphism.

Corollary I.10. Let $G$ be Noetherian. Then every $h \in H$ with $\sigma(h), \tau(h) \in[i]$ for some $i \in I$ is an isomorphism. In particular, if $[i]$ is minimal with respect to $\preceq$, then every nonzero $h \in H$ with $\sigma(h) \in[i]$ is an isomorphism.

Proof. Since the mappings in $\mathscr{H}$ are all epimorphisms, the result follows from the above theorem and the well-known theorem that an onto endomorphism of a Noetherian module is an automorphism.

For future use we note that in the case when $\mathscr{H}=$ Hom, if $h \in H$ is an isomorphism, then $h^{-1} \in H$. Hence the above corollary says

Corollary I.11. Let $G$ be Noetherian, let $(\mathscr{C}, \mathscr{H})$ be a matched pair on $G$, and let [i] be minimal. If $h \in H$ is nonzero with $\sigma(h) \in[i]$, then $h^{-1} \in H$.

Another easy result in this vein is 
Theorem I.12. If $C_{i} \in \mathscr{C}$ is minimal in $G$, then [i] is minimal and every $h \in H$ with $\sigma(h), \tau(h) \in[i]$ is an isomorphism.

Convention. Henceforth, for convenience of exposition we take $0 \in I$ and $C_{0}=$ $\{0\}$. Note that $[0]=\{0\}$.

We conclude this section by characterizing when $M(\mathscr{C}, \mathscr{C})$ is a ring. From this, we note that, in general, $M(\mathscr{C}, \mathscr{H})$ is not a ring.

Theorem I.13. Let $(\mathscr{C}, \mathscr{H})$ be a matched pair on $G$. The following are equivalent:

(1) $M(\mathscr{C}, \mathscr{H})$ is a ring.

(2) For each nonzero $h \in H, \sigma(h)=\tau(h)$.

(3) $\forall i \in I \backslash\{0\},[i]$ is minimal and $[i]=\{i\}$.

Proof. From the definitions, one observes that (2) and (3) are equivalent, so we show (1) and (2) are equivalent. Let $M(\mathscr{C}, \mathscr{H})$ be a ring, and assume there is $0 \neq h \in H$ with $h: C_{j} \rightarrow C_{i}, i \neq j$. Define $t \in M$ by $t_{j}=h$ and $t_{i}=0$, $i \neq j$. Since $M$ is a ring, $\varepsilon^{i}\left(t+\varepsilon^{j}\right)=\varepsilon^{i} t+\varepsilon^{i} \varepsilon^{j}=t$. Let $\tau\left(t_{j}+\left(\varepsilon^{j}\right)_{j}\right)=l$. Then for each $x \in C_{j},\left[\varepsilon^{i}\left(t+\varepsilon^{j}\right)\right](x)=\left(\varepsilon^{i}\right)_{l}(h(x)+x)=t_{j}(x)=h(x)$. If $l \neq i$, we get $0=h(x)$, which is a contradiction since $0 \neq h$. If $l=i$, we have $h(x)+x=h(x)$, so $x=0$, again a contradiction since $C_{j} \neq\{0\}$. Hence if $M(\mathscr{C}, \mathscr{H})$ is a ring, we must have $\sigma(h)=\tau(h)$, for each $0 \neq h \in H$. Conversely, we note that if $\sigma(h)=\tau(h)$ for each nonzero $h \in H$, then $H_{i}$ is a subring of $\operatorname{End}_{R} C_{i}$ and $M(\mathscr{C}, \mathscr{H})=\times_{I} H_{i}$ with pointwise addition and multiplication, i.e., $M(\mathscr{C}, \mathscr{H})$ is a ring.

\section{LEFT IDEALS}

Recall that a left ideal $L$ of an arbitrary near-ring $N$ is strictly minimal (strictly maximal) if $L$ is minimal (maximal) as a left invariant subgroup. In this section we identify some minimal left ideals and strictly minimal left ideals of $M=M(\mathscr{C}, \mathscr{H})$.

For any subset $J$ of $I$, the set $A(J):=\left\{s \in M \mid s_{j}=0\right.$ if $\left.j \in J\right\}$ is a left ideal of $M$. In particular, every $M \varepsilon^{i}$ is a left ideal since $M \varepsilon^{i}=A(I \backslash\{i\})$. We investigate when $M \varepsilon^{i}$ is minimal, and to this end we transfer the inclusion partial order on $\mathscr{C}$ to $I$, writing $\leq$ for the resulting partial order on $I$.

Theorem II.1. If $[i]$ is minimal with respect to $\preceq$ and there exists $j \in[i]$ such that $j \not \leq i$, then $M \varepsilon^{i}$ is a minimal left ideal of $M$.

Proof. Let $L$ be a nonzero left ideal of $M$ with $L \subseteq M \varepsilon^{i}$, and take $0 \neq s \in L$. Then $s_{i} \neq 0$. We claim that without loss of generality we may assume $\tau\left(s_{i}\right)=j$. For, since [i] is minimal, $\tau\left(s_{i}\right) \in[i]$, so there is some $h \in H$ such that $\tau\left(h s_{i}\right)=j$. If $t \in M$ is such that $(t)_{\tau\left(s_{i}\right)}=h$, then $t s \in L$ and $\tau\left((t s)_{i}\right)=j$. Now let $x \in M$ be arbitrary with $s_{i}+x_{i}=e_{i}$. Therefore, $\tau\left(x_{i}\right) \neq i$; otherwise, for each $c \in C_{i}, s_{i}(c)=c-x_{i}(c) \in C_{i}$, which implies $C_{j} \subseteq C_{i}$, i.e., $j \leq i$. Let $y \in M$ be defined by $y_{i}=e_{i}$ and $y_{k}=0$ for $k \neq i$. Then $z:=y(s+x)-y x \in L$ and $z_{i}=y_{i}\left(s_{i}+x_{i}\right)-y_{\tau\left(x_{i}\right)} x_{i}=e_{i}$, so $z=\varepsilon^{i}$. But this means $\varepsilon^{i} \in L$ and $L=M \varepsilon^{i}$.

Corollary II.2. If $[i]$ is minimal with respect to $\preceq$, then $M \varepsilon^{k}$ is a minimal left ideal of $M$ for all but (possibly) one $k \in[i]$. 
Proof. If there is more than one element in [i], then for at most one $k \in[i]$ can we have that $j \leq k$ for all $j \in[i]$.

To illustrate the above, let $G$ be any torsion free abelian group regarded as a $\mathbb{Z}$-module, and consider the matched pair $\left(\mathscr{C}_{G}(\mathbb{Z}), \mathrm{Hom}\right)$. Then $I$ has just two equivalence classes, $[0]=\{0\}$ and $I \backslash\{0\}$, the latter being minimal. If $G$ is the infinite cyclic group, then $G \in \mathscr{C}_{G}(\mathbb{Z})$, say $G=C_{k}$. Then $j \leq k$ for all $j \in I \backslash\{0\}$, but for each $j \in I \backslash\{0\}, j \neq k$ we have $k \not \leq j$, so $M \varepsilon^{j}$ is minimal for each $j \in I \backslash\{0\}, j \neq k$. We note that, in this case, it turns out that $M \varepsilon^{k}$ is also minimal (see Theorem 2.5). By contrast, let $G:=Q$ and $R:=\mathbb{Z}$, and consider the pair $(\mathscr{C}, \mathscr{H})$ where $\mathscr{C}:=\left\{C_{0}:=\{0\}, C_{1}:=Q\right\}$ and $\mathscr{H}:=\{0, \mathbb{Z}\}$. Then $M(\mathscr{C}, \mathscr{H})=M \varepsilon^{1} \cong \mathbb{Z}$, so $M \varepsilon^{1}$ is not minimal; in fact, $M$ has no minimal left ideal. Note here that $[i]=\{i\}$ for $i \in I$; so if $[i]=\{i\}$ and $[i]$ is minimal, it need not be the case that $M \varepsilon^{i}$ is minimal.

Theorem II.3. The left ideal $M \varepsilon^{i}$ is strictly minimal if and only if $h \in H_{i}$, $h \neq 0$, implies $h^{-1} \in H$.

Proof. This follows from the fact that for $s \in M, M s$ will be properly contained in $M \varepsilon^{i}$ if and only if $s=s \varepsilon^{i}$ and there is no $t \in M$ such that $t s=\varepsilon^{i}$, i.e., such that $(t s)_{i}=t_{\tau\left(s_{i}\right)} s_{i}=e_{i}$.

Since $M=A(\{i\}) \oplus M \varepsilon^{i}$, we have the following dual result about maximal left ideals.

Theorem II.4. Let [i] be minimal with respect to $\preceq$.

(a) If there is $j \in[i]$ with $j \not i$, then $A(\{i\})$ is a maximal left ideal of $M$.

(b) $A(\{k\})$ is maximal for all but (possibly) one $k \in[i]$. Moreover, the left ideal $A(\{j\})$ is strictly maximal if and only if $h \in H_{j}, h \neq 0$, implies $h^{-1} \in H$.

Combining Theorem II.3, the last part of Theorem II.4, and Corollary I.11 we obtain

Theorem II.5. Let $G$ be Noetherian and $(\mathscr{C}, H)$ be a matched pair on $G$. If [i] is minimal with respect to $\preceq$, then $M \varepsilon^{j}$ is strictly minimal and $A(\{j\})$ is strictly maximal for each $j \in[i]$.

Recall that a near-ring $N$ is 2-semisimple provided the intersection of all strictly maximal ideals of $N$ is $\{0\}$, i.e., if $J_{2}(N)=\{0\}$. When $G$ is Noetherian and [i] is minimal for each $i \in I$, then $J_{2}(M) \subseteq \bigcap_{i \in I} A(\{i\})=\{0\}$. Thus we have

Corollary II.6. Under the hypothesis of Theorem II.5, if [i] is minimal with respect to $\preceq$ for each nonzero $i \in I$, then $M$ is 2-semisimple.

\section{TWO-SIDED IDEALS}

As noted above, the $A(J)$ are obvious examples of left ideals in $M(\mathscr{C}, \mathscr{H})$. In this section we focus on two-sided ideals. We first determine when the $A(J)$ will be two-sided. We then investigate conditions under which $M=M(\mathscr{C}, \mathscr{H})$ is a simple near-ring.

We say a nonempty set $J \subseteq I$ is a sink if $\sigma(h) \in J$ implies $\tau(h) \in J$. Note that both $\{0\}$ and $I$ are sinks which we refer to as the trivial sinks. 
Theorem III.1. For $\varnothing \neq J \subseteq I$, the left ideal $A(J)$ is a two-sided ideal if and only if $J$ is a sink.

Proof. Let $J$ be a sink. We show $A(J)$ is a right ideal. If $s \in A(J)$ and $t \in M$, then for $j \in J,(s t)_{j}=s_{k} t_{j}$ where $\tau\left(t_{j}\right)=k$. Since $j \in J$, we have $k \in J$ and thus, since $s \in A(J), s_{k}=0$. On the other hand, if $J \neq \varnothing$ is not a sink, then there exists $h \in H$ with $j:=\sigma(h)$ and $k:=\tau(h)$ with $k \notin J$. Let $s \in A(J)$ with $s_{k}=e_{k}$, and let $t \in M$ with $t_{j}=h$. Then $(s t)_{j}=s_{k} t_{j}=h \neq 0$, so $s t \notin A(J)$.

In light of this result it is of some importance to determine the sinks in $I$. The next result gives some information in this regard.

Theorem III.2. (a) If $J$ is any nonempty subset of $I$, then $\Sigma(J):=\{\tau(h) \mid h \in H$ and $\sigma(h) \in J\}$ is a sink called the sink generated by $J$.

(b) If $K:=\bigcup\{[i] \mid[i] \preceq[j]$ for some $j \in J \subseteq I\}$, then $K=\Sigma(J)$.

(c) If $\mathscr{A}$ is any class of $R$-modules and $\mathscr{B} \subseteq \mathscr{A}$, then $\Sigma(\mathscr{B}):=\left\{i \in I \mid C_{i} \in\right.$ $\left.\mathscr{C}_{G}(\mathscr{B})\right\}$ is a sink with regard to any matched pair $\left(\mathscr{C}_{G}(\mathscr{A}), \mathscr{H}\right)$.

(d) If $\mathcal{J}$ is any family of sinks in $I$, then $\bigcup \mathscr{J}$ and $\cap \mathcal{J}$ are also sinks.

Proof. (a) Suppose $h \in H$ with $\sigma(h) \in \Sigma(J)$. Thus there exists $h^{\prime} \in H$ with $\sigma\left(h^{\prime}\right) \in J$ and $\tau\left(h^{\prime}\right)=\sigma(h)$. But then $h h^{\prime} \in H$ and $\sigma\left(h h^{\prime}\right)=\sigma\left(h^{\prime}\right) \in J$. Hence $\tau(h)=\tau\left(h h^{\prime}\right) \in \Sigma(J)$ as desired.

(b) If $i \in \Sigma(J)$, then there is some $h \in H$ with $\sigma(h) \in J$ and $\tau(h)=i$. Consequently, $[i] \preceq[\sigma(h)]$, so $i \in K$. Conversely, if $i \in K$, then $[i] \preceq[j]$, for some $j \in J$, which in turn implies that there exists $h \in H$ with $\sigma(h)=j$ and $\tau(h)=i$. Hence $i \in \Sigma(J)$.

(c) If $\sigma(h) \in \Sigma(\mathscr{B})$, then $\tau(h) \in \Sigma(\mathscr{B})$ since $h\left(C_{\sigma(h)}\right) \in \mathscr{C}_{G}(\mathscr{B})$.

(d) This is clear.

We apply this theorem to obtain some specific instances of sinks.

Corollary III.3. The following are sinks for any matched pair $(\mathscr{C}, \mathscr{H})$ on $G$ :

(a) $[i] \cup\{0\}$ if $[i]$ is minimal.

(b) $\left\{i \mid C_{i}\right.$ has property $\left.\mathscr{P}\right\}$, where $\mathscr{P}$ is any property preserved by homomorphisms, e.g., the property of begin simple or $\{0\}$, singular, finitely generated, cyclic, Noetherian, and Artinian.

Proof. Part (a) follows from III.2(b), and part (b) follows from III.2(c).

Another way of getting sinks in the case $(\mathscr{C}$, End) is given by

Theorem III.4. Let $F \leq G$ be a fully invariant submodule. Then $\left\{i \in I \mid C_{i} \subseteq F\right\}$ is a sink with respect to the matched pair $(\mathscr{C}$, End).

This result, whose straightforward proof is omitted, shows, for example, that there are sinks associated with $\operatorname{Rad} G$ and $\operatorname{Soc} G$ and with the singular submodule $Z(G)$ of $G$.

We now turn to simplicity of $M(\mathscr{C}, \mathscr{H})$. From the above we know if there are two or more nonzero classes in $I / \sim$, then $M$ cannot be simple. However, the existence of just one nonzero class does not guarantee simplicity, as the example with $G=Q$ and $R=\mathbb{Z}$ discussed after Corollary II.2 shows. We start with a decomposition result.

Lemma III.5. Suppose $I=[0] \cup\left(\bigcup_{k=1}^{n}\left[i_{k}\right]\right)$ where each $\left[i_{k}\right]$ is minimal. Then $M=\sum_{k=1}^{n} \bigoplus M_{k}$ where $M_{k}:=A\left(I \backslash\left[i_{k}\right]\right)$ is an ideal. 
Proof. Note that $I \backslash\left[i_{k}\right]$ is a sink for each $k, M=\sum_{k=1}^{n} M_{k}$, and $M_{k} \cap$ $\left(\sum_{k \neq l} M_{l}\right)=\{0\}$

Recall that $\mathscr{S}$ is the class of simple $R$-modules. We now consider the case $\left(\mathscr{C}_{G}(\mathscr{S})\right.$, Hom $)$.

Theorem III.6. If $G$ is Noetherian with $\operatorname{Soc} G \neq\{0\}$, then $M\left(\mathscr{C}_{G}(\mathscr{S})\right.$, Hom $)$ is a direct sum of a finite number of ideals, each of which is invariantly simple as a near-ring.

Proof. Since $G$ is Noetherian, there are only finitely many isomorphism types of simple $R$-modules represented in $\mathscr{C}_{G}(\mathscr{S})$. Each isomorphism class gives rise to a class in $I / \sim$ which is minimal with respect to $\preceq$. Since the union of these classes together with [0] is $I$, we can apply the preceding lemma to obtain a decomposition of $M$ into ideals. To complete the proof, it suffices to show if $I=[0] \cup[i]$, where $C_{j}$ is simple for $j \in[i]$, then $M\left(\mathscr{C}_{G}(\mathscr{S})\right.$, Hom $)$ is invariantly simple. Since $G$ is Noetherian, so is $F:=\operatorname{Soc} G$, and therefore $F$ is the direct sum of some of the $C_{j}$. For ease of notation, we let $F=\sum_{i=1}^{n} \oplus C_{i}$. Denote by $\eta^{i}: F \rightarrow C_{i}, i=1,2, \ldots, n$, the projection onto $C_{i}$. Thus $\sum_{i=1}^{n} \eta^{i}=1_{F}$. Also, $\eta^{i}$ represents an element of $M$ by defining $\left(\eta^{i}\right)_{l}:=$ $\left(\eta^{i} \mid C_{l}\right), l \in I$, and in this context, $\sum_{i=1}^{n} \eta^{i}=e$, the identity in $M$. Now let $s \in M, s \neq 0$, with, say, $s_{j} \neq 0$. From Corollary I.11, $s_{j}^{-1} \in H$. Therefore, if $t \in M$ is such that $t_{\tau\left(s_{j}\right)}=s_{j}^{-1}$, then $\varepsilon^{j}=t s \varepsilon^{j} \in\langle s\rangle$, the invariant subgroup of $M$ generated by $s$. But from $\varepsilon^{j}$ we can easily construct $\varepsilon^{i}, i=1,2, \ldots, n$, by left and right multiplications, again using Corollary I.11. Now $\eta^{i}=\varepsilon^{i} \eta^{i}$ for $i=1,2, \ldots, n$, because, since $C_{i}$ is simple, $\tau\left(\left(\eta^{i}\right)_{l}\right)$ is either $i$ or 0 , and therefore $e=\sum_{i=1}^{n} \eta^{i} \in\langle s\rangle$. This completes the proof.

Since every simple submodule of an $R$-module $G$ is cyclic, we have

Corollary III.7. If $G$ is a semisimple, Noetherian module, then $M\left(\mathscr{C}_{G}(R)\right.$, Hom) is a direct sum of a finite number of ideals, each of which is invariantly simple as a near-ring.

A special case is the following

Corollary III.8. If $V$ is a finite-dimensional vector space over a division ring, then $M\left(\mathscr{C}_{V}(D), \mathrm{Hom}\right)$ is invariantly simple.

The above result is not true for an infinite-dimensional vector space, $V$, over a division ring $D$. In fact, if we define, for $f \in M, \mathscr{R}(f):=\operatorname{Span}\left\{f\left(C_{i}\right) \mid C_{i} \in\right.$ $\mathscr{C}\}$ and let $K:=\{f \in M \mid \operatorname{dim} \mathscr{R}(f)<\infty\}$, then $K$ is an invariant subgroup of $M,\{0\} \subsetneq K \subsetneq M$ However, from the next result, which has wide applicability, we will find that in this case $M\left(\mathscr{C}_{V}(D)\right.$, Hom) is simple.

Theorem III.9. Let $(\mathscr{C}, \mathscr{H})$ be a matched pair on $G$ with the following properties:

(i) There is only one nonzero class [i].

(ii) If $h \in H$ is nonzero, then $h^{-1} \in H$.

(iii) There is some $F \leq G$ such that $\left|\left\{i \in I \mid C_{i} \subseteq F\right\}\right|=|I|$.

(iv) There exists a $C \in \mathscr{C}$ with $C \nsubseteq F$.

Then $M(\mathscr{C}, \mathscr{H})$ is a simple near-ring. 
Proof. For convenience we let $C_{1}:=C$ and $J:=\left\{i \in I \mid C_{i} \subseteq F\right\}$. From (iii) there is a bijection between $I$ and $J$, so without loss of generality we let $\Phi: I \rightarrow J$ be a bijection with $\Phi(0)=0$. Suppose $s$ is a nonzero element of $M$. We show that the ideal generated by $s$, which we denote by $T$, is $M$. We first show that $\varepsilon^{1} \in T$. Indeed, if $s_{i} \neq 0$, then we know there exist $u, v \in M$ with $\tau\left(v_{1}\right)=i$ and $v_{j}=0$ for $j \neq 1$ and $u_{\tau\left(s_{i}\right)}=v_{1}^{-1} s_{i}^{-1}, u_{j}=0$ otherwise. Then $u s v=\varepsilon^{1}$. Next, let $t \in M$ be such that $\tau\left(t_{i}\right)=1$ if $i \neq 0$. Hence $\varepsilon^{1} t=t$. Now choose $x \in M$ so that $\tau\left(t_{i}+x_{i}\right)=\Phi(i)$. We claim that $\tau\left(x_{i}\right) \notin J$ for all $i \neq 0$. To establish this for a particular $i$, let $g \in C_{i}$ be such that $t_{i}(g) \notin F$, which is possible because $C_{1} \subsetneq F$. Then $\left(t_{i}+x_{i}\right)(g)=t_{i}(g)+x_{i}(g) \in C_{\Phi(i)} \subseteq F$. This implies $x_{i}(g) \notin F$, which proves our assertion. Finally, choose $y \in M$ with $y_{j}=\left(t_{\Phi^{-1}(j)}+x_{\Phi^{-1}(j)}\right)^{-1}$ if $0 \neq j \in J$ and $y_{j}=0$ if $j \notin J$ or $j=0$. Then $y x=0$ and $y(t+x)=e$, so $e=y(t+x)-y x \in T$, i.e. $T=M$.

We remark that Theorem I.7 follows from Theorem III.9. Further we have Corollary III.10. Let $V$ be a vector space over a division ring $D$. Then $M\left(\mathscr{C}_{V}(D)\right.$, Hom $)$ is a simple near-ring.

Proof. The finite-dimensional case is that of Corollary III.8. For the infinitedimensional case, let $\mathscr{B}=\left\{b_{k}\right\}, k \in \mathscr{K}$, be a basis for $V$. Then the cardinality of the set of cyclic submodules in $V^{\prime}:=\operatorname{Span}\left(\mathscr{B} \backslash\left\{b_{k_{0}}\right\}\right)$ is the same as that of the set of all cyclic submodules in $V$. So, by applying Theorem I.12 and choosing $F:=V^{\prime}, C:=b_{k_{0}} D$, we see that the hypotheses of Theorem III.9 are satisfied. Thus $M\left(\mathscr{C}_{V}(D)\right.$, Hom $)$ is simple.

As a further application of Theorem III.9 we let $K$ be any infinite field and let $R:=K[x]$ and $G:=K[x]$. With $\mathscr{C}:=\mathscr{C}_{G}(R)$ and $\mathscr{H}:=$ Hom, it is clear that (i), (ii), and (iii) of Theorem III.9 are satisfied if we take $F:=x K[x]$. Note that for each $\alpha \in K,\left(x^{2}+\alpha x\right) K[x] \subseteq F$, but $K[x] \nsubseteq F$.

We wish to characterize when $M=M\left(\mathscr{C}_{G}(R)\right.$, Hom $)$ is a simple near-ring. As we have noted above, if $M$ is simple, then there is only one nonzero class with respect to $\sim$, i.e., $|I / \sim|=2$. So, we focus now on $|I / \sim|=2$. In certain situations this condition is also sufficient.

Theorem III.11. Let $\mathscr{C}=\mathscr{C}_{G}(R), \mathscr{H}=\mathrm{Hom}$, and suppose $G$ has finite length. Then the following are equivalent:

(1) $M$ is simple.

(2) $M$ is invariantly simple.

(3) $|I / \sim|=2$.

In this case, $G$ is semisimple.

Proof. We know (2) $\Rightarrow(1)$ and $(1) \Rightarrow(3)$, so it remains to show (3) $\Rightarrow(2)$. To this end let $C_{j} \in \mathscr{C}$. Since $\mathscr{C}=\mathscr{C}_{G}(R), C_{j}$ is a cyclic $R$-module. We show each $C_{j}$ is simple. Assume the contrary. Then there is a cyclic submodule $C_{i}$ of $C_{j}, C_{i} \subsetneq C_{j}$. Now, $C_{i} \in \mathscr{C}$ and $|I / \sim|=2$ implies there exists $h \in H, h: C_{j} \rightarrow C_{i}$. Moreover, since $G$ is of finite length, $G$ is Noetherian, so $h^{-1} \in H$. But then, considering $h \mid C_{i}$, we get $h\left(C_{i}\right)=C_{k} \subsetneq C_{i}$, otherwise $\operatorname{Ker}(h) \neq\{0\}$. In this manner we get an infinite descending chain in $G$, contrary to $G$ being of finite length. Since each cyclic is simple, $\operatorname{Soc} G \neq\{0\}$, so now as in the proof of Theorem III.6, we get that $M$ is invariantly simple.

To show that $G$ is semisimple, let $a \in G$. If $G=a R$, then we are finished. If not, there exists $b \in G \backslash a R$. Then $a R \cap b R=\{0\}$ since they are both simple. 
If $G=a R \oplus b R$, then again we are finished. If not, we find $c \in G \backslash a R \oplus b R$. This process must end since $G$ is of finite length.

Corollary III.12. If $R$ is an Artinian ring and $G$ is finitely generated, then the following are equivalent:

(1) $M$ is simple.

(2) $M$ is invariantly simple.

(3) $|I / \sim|=2$.

Further, if $G$ is a faithful $R$-module, then $R$ is a semisimple ring.

Proof. Since $R$ is Artinian, $R$ is Noetherian, and $G$ is finitely generated, $G$ has finite length. Thus the first sentence follows from Theorem III.11. Suppose now $G$ is faithful. For each $g \in G \backslash\{0\}, g R$ is simple, so $r_{R}(g)=\{r \in$ $R \mid g r=0\}$ is a maximal right ideal of $R$. Therefore, $J(R) \subseteq \bigcap_{g \in R} r_{R}(g)=$ $A(G)=\{0\}$, hence the result.

We remark that Theorems I.6 and I.7 show that the above corollary is not true if we take $R$ to be Noetherian. We are thus led to the following problem which remains open.

Problem. Let $G$ be Noetherian with matched pair $\left(\mathscr{C}_{G}(R)\right.$, Hom $)$ and $|I / \sim|$ $=2$. Is $M\left(\mathscr{C}_{G}(R)\right.$, Hom $)$ a simple near-ring?

We conclude this section and the paper with one further special case. We start here with a few remarks which provide a summary of the above, indicating the present status of the problem. First, we note that all of the nonzero $C_{i} \in \mathscr{C}$ are isomorphic, and if $h: C_{i} \rightarrow C_{j}$ is any isomorphism, then $h \in H$. Moreover, if $h \in H, h \neq 0$, then $h^{-1} \in H$.

Suppose any $C \in \mathscr{C}$ is minimal; then it is simple, and hence every $C \in \mathscr{C}$ is simple. So this is the semisimple case, and since $G$ is Noetherian, $G$ is the direct sum of finitely many simple submodules. This case was handled in Theorem III.6. So, henceforth, we assume every nonzero $C \in \mathscr{C}$ contains an infinite descending chain of elements of $\mathscr{C}$. If any one (and therefore all) of them contains $|I|$ elements of $\mathscr{C}$, then we can apply Theorem III.9. We therefore assume that no element of $\mathscr{C}$ contains $|I|$ elements of $\mathscr{C}$. This implies that $G$ is not cyclic, so every cyclic is proper.

Since $G$ is Noetherian, $G$ is the sum (albeit not necessarily direct) of finitely many elements of $\mathscr{C}$. For convenience we take $G=\sum_{i=1}^{n} C_{i}$. Let $J_{i}:=$ $\left\{j \in I \mid C_{j} \subseteq C_{i}\right\}, i=1,2, \ldots, n$, and let $C_{j_{i}}$ be chosen such that $j_{i} \notin J_{i}$, i.e., $C_{j_{i}} \nsubseteq C_{i}$.

Let $T$ be any nonzero ideal of $M$. Then, for each $l \in I, \varepsilon^{l} \in T$. In fact, let $0 \neq s \in T$. For some $i \in I, s_{i} \neq 0$. Furthermore, there exists $h \in H$, $h: C_{l} \rightarrow C_{i}$. We let $u \in M$ be defined by $u_{l}=h$ and $u_{j}=0, j \neq l$, and let $v \in M$ be defined by $v_{\tau\left(s_{i}\right)}=h^{-1} s_{i}^{-1}$ and 0 otherwise. Then $v s u \varepsilon^{l} \in T$ and $v s u \varepsilon^{l}=\varepsilon^{l}$. Note that here we have used only the fact that $T$ is an invariant subgroup.

Now, for $i=1,2, \ldots, n$ define $\delta^{i} \in M$ by $\left(\delta^{i}\right)_{j}=e_{j}$ if $j \in J_{i}$ and 0 otherwise. We show $\delta^{i} \in T$. Indeed, let $s \in M$ with $\tau\left(s_{j}\right)=j_{i}$ if $j \in J_{i}$ and 0 otherwise. Then $\varepsilon^{j_{i}} s=s \in T$ since $\varepsilon^{j_{i}} \in T$. Now choose $x \in M$ such that $s_{j}+x_{j}=e_{j}$ if $j \in J_{i}$ and 0 otherwise. If $c \in C_{j}$ is such that $s_{j}(c) \notin C_{i}$ (which is possible because $s_{j}\left(C_{j}\right)=C_{j_{i}} \nsubseteq C_{i}$ ), then $s_{j}(c)+x_{j}(c)=c$. Therefore, $x_{j}(c) \notin C_{i}$, i.e., $\tau\left(x_{j}\right) \notin J_{i}$. From this we get $\delta^{i}(s+x)-\delta^{i} x=\delta^{i} \in T$. 
We now make an additional assumption, namely, if $C_{i}, C_{j} \in \mathscr{C}$ with $C_{i}=$ $g_{i} R$ and $C_{j}=g_{j} R$, then there exists $h: C_{i} \rightarrow C_{j}$ with $h\left(g_{i}\right)=g_{j}$. From this, we show $e \in T$. Let $K:=\left\{g_{i} \mid i \in I\right\}$ be a set of generators for $\mathscr{C}$, i.e., $C_{i}=g_{i} R$. Let $j \in I, j \neq 0$, and suppose $g_{j}=f_{j 1}+\cdots+f_{j n}$, where $f_{j i} \in C_{i}$. Each $f_{j i}$ is a generator for a $C_{j i}$, although we do not necessarily have $f_{j i} \in K$. However, we do have $j i \in J_{i}$. Let $h_{j i} \in H$ be that isomorphism of $C_{j}$ onto $C_{j i}$ which takes $g_{j}$ to $f_{j i}$, and let $\left(\gamma^{i}\right)_{j}=h_{j i}$, for each $j \in I, j \neq 0$. Then $\delta^{i} \gamma^{i}=\gamma^{i} \in T$. We claim that $\sum_{i=1}^{n} \gamma^{i}=e$. Indeed, $\left(\sum_{i=1}^{n} \gamma^{i}\right)_{j}\left(g_{j}\right)=$ $\left(h_{j 1}+\cdots+h_{j n}\right)\left(g_{j}\right)=f_{j 1}+\cdots+f_{j n}=g_{j}$. Hence, $e \in T$, and consequently $M$ is simple. We have established

Theorem III.13. Let $G$ be a noncyclic Noetherian $R$-module with matched pair $\left(\mathscr{C}_{G}(R)\right.$, Hom) satisfying $|I / \sim|=2$. If for each $C_{i}, C_{j} \in \mathscr{C}, C_{i}=g_{i} R$, $C_{j}=g_{j} R$, there exists $h: C_{i} \rightarrow C_{j}$ with $h\left(g_{i}\right)=g_{j}$, then $M\left(\mathscr{C}_{G}(R)\right.$, Hom $)$ is a simple near-ring.

\section{ACKNOWLEDGMENT}

This paper was written while the second author was a visiting professor at Texas A \& M University in 1991. He wishes to express his gratitude for the financial assistance and hospitality provided by Texas A \& $M$.

\section{REFERENCES}

1. F. W. Anderson and K. R. Fuller, Rings and categories of modules, Graduate Texts in Math., vol. 13, Springer-Verlag, New York, Heidelberg, and Berlin, 1973.

2. P. Fuchs, C. J. Maxson, and G. Pilz, On rings for which homogeneous maps are linear, Proc. Amer. Math. Soc. 112 (1991), 1-7.

3. C. J. Maxson, Piecewise endomorphisms of PID-modules, Results in Math. 18 (1990), 125-132.

4. C. J. Maxson and A. P. J. van der Walt, Homogeneous maps as piecewise endomorphisms, Comm. Algebra 20 (1992), 2755-2776.

5. J. D. P. Meldrum, Near-rings and their links with groups, Pitman Res. Notes Math. Ser., vol. 134, Longman Sci. Tech., Harlow, 1985.

6. G. F. Pilz, Near-rings, 2nd ed., North-Holland, Amsterdam, 1983.

Department of Mathematics, Texas A \& M University, College Station, Texas 77843

E-mail address: cjmaxson@math. tamu.edu

Department of Mathematics, University of Stellenbosch, 7600 Stellenbosch, South AFrica

E-mail address: apjwCsunvax.sun.ac.za 